\title{
OPTIMAL CONTROL AND SEMICONTINUOUS VISCOSITY SOLUTIONS
}

\author{
E. N. BARRON AND R. JENSEN
}

(Communicated by Kenneth R. Meyer)

\begin{abstract}
We study the value function for an optimal control problem with upper semicontinuous terminal data. We prove that the upper semicontinuous envelope of the value function is the unique semicontinuous viscosity solution of the Bellman equation and that it coincides with the value function obtained when using relaxed controls.
\end{abstract}

\section{INTRODUCTION AND SUMMARY}

In [2] we introduced an extension of the theory of viscosity solutions for Hamilton-Jacobi equations of the form $\Lambda(u) \equiv u_{t}+H\left(t, x, D_{x} u\right)=0$ if $(t, x) \in(0, T) \times R^{n}$ and $u(T, x)=g(x)$. The classical theory in which $g$ is assumed to be continuous was initiated in the pioneering paper of Crandall and Lions [4]. Our extension is to allow semicontinuous terminal data $g$ but we require the Hamiltonian $H(t, x, p)$ to be convex (for upper semicontinuous $g$ ) in $p$. For lower semicontinuous terminal data we require concavity. This convexity assumption is satisfied in problems of optimal control of ordinary differential equations, where we are dealing with the Bellman equation.

From now on we discuss only the upper semicontinuous (u.s.c) case with the lower semicontinous case being entirely analogous.

The problem with u.s.c data, of course, is that we can only deal with u.s.c candidate functions as solutions to the Bellman equation since it is known that discontinuities propagate in first order problems. This leads to the difficulty that the definition of viscosity supersolution is vacuuous in general because it requires the existence of a smooth function, say $\phi$ for which $u-\phi$ achieves a minimum at some point and $\Lambda(\phi) \leq 0$ at this point. There is no problem with the definition of viscosity subsolution, which requires that $u-\phi$ achieve a maximum and then $\Lambda(\phi) \geq 0$ at the max point. In [2] we defined $u$ to be

Received by the editors April 7, 1990.

1980 Mathematics Subject Classification (1985 Revision). Primary 49C20, 35D99.

Key words and phrases. Optimal control, semicontinuous data, semicontinuous viscosity solutions, relaxed controls.

Barron was partially supported by grant No. AFOSR-86-0202 from the Air Force Office for Scientific Research. Jensen was also supported by a grant from the National Science Foundation. 
an u.s.c viscosity solution if $u$ is an u.s.c function and if $u-\phi$ achieves a maximum then $\Lambda(\phi)=0$ at the max point. In [2] we were able to establish that this uniquely characterizes a u.s.c viscosity solution.

In this paper our goal is to establish that for an optimal control problem with u.s.c terminal data, the upper semicontinuous envelope of the usual value function is in fact the u.s.c viscosity solution of the Hamilton-Jacobi-Bellman equation for the problem. This proves that taking the u.s.c (lower semicontinuous) envelope is the right thing to do in maximization (minimization) optimal control problems. The implication is that it is also the right thing to do for differential games, i.e., for problems with nonconvex, nonconcave hamiltonians. This gives further justification of the approach initiated by Ishii [5] in which he introduced the extension of viscosity theory to possibly discontinuous solutions by using upper and lower semicontinuous envelopes. However, it is important to note that most previous work, including [5], assumed that the data is continuous.

The method of proof of our result is to prove that the value function using relaxed controls is the u.s.c viscosity solution. Then we directly prove that the upper semicontinuous envelope of the original value function and the relaxed value function coincide.

\section{The MAIN RESULTS}

We use the simplest model for optimal control, i.e., the Mayer problem on the finite time interval $[0, T]$. Since problems with running costs can be reduced to this case, this is more general than it seems. The problem dynamics are given by

$$
\begin{gathered}
d \xi / d \tau=f(\tau, \xi(\tau), \eta(\tau)) \quad \text { if } t<\tau \leq T, \\
\xi(\tau)=x \in R^{n} .
\end{gathered}
$$

The control functions $\eta$ are chosen from the class of functions

$$
Y[t, T]=\{\eta:[t, T] \rightarrow Y \mid \eta \text { is Lebesgue measurable }\},
$$

where $Y$ is a compact subset of some $R^{p}, p \geq 1$. The objective is to maximize some function $g$ of the terminal state $\xi(T)$. We define the value function $V:[0, T] \times R^{n} \rightarrow R^{1}$ as follows:

$$
V(t, x)=\sup _{\eta \in Y[t, T]} g(\xi(T)) .
$$

We make the following assumptions regarding the given functions $f$ and $g$ :

(A) $f:[0, T] \times R^{n} \times Y \rightarrow R^{n}$ is continuous in all arguments and there is $a$ constant $K>0$ such that $|f(t, x, y)| \leq K,(t, x, y) \in[0, T] \times R^{n} \times Y$, and

$$
|f(t, x, y)-f(t, z, y)| \leq K|x-z| \quad \forall(t, y) \in[0, T] \times Y \text { and } x, z \in R^{n} \text {. }
$$

$g: R^{n} \rightarrow R^{1}$ is bounded above and upper semicontinuous. 
The assumption (A) is sufficient to guarantee that for each control $\eta \in$ $Y[t, T]$ there is a unique trajectory $\xi(\cdot)$ on the interval $[t, T]$ with $\xi(t)=x$.

For later use we define the relaxed control problem as follows. Let $M(Y)$ denote the space of Radon probability measures on the set $Y$. The space $M(Y)$ is identified with the dual space $C(Y)^{*}$ of the space $C(Y)$ of continuous functions on $Y$, and $M(Y)$ has the weak-* topology of $C(Y)^{*}$. The space of relaxed controls, denoted by $Y^{*}[t, T]$, is the space of measureable maps $\mu:[t, T] \rightarrow M(Y)$. A relaxed control at time $s \in[0, T]$ is denoted by $\mu_{s}$. Given a relaxed control $\mu$, a relaxed trajectory $\xi^{*}(\cdot)$ on $[t, T]$ is the solution of

$$
\xi^{*}(\tau)=x+\int_{t}^{T} \int_{Y} f\left(s, \xi^{*}(s), y\right) d \mu_{s}(y) d s .
$$

We define the relaxed value function $V^{*}:[0, T] \times R^{n} \rightarrow R^{1}$ as follows:

$$
V^{*}(t, x)=\sup _{\mu \in Y^{*}[t, T]} g\left(\xi^{*}(T)\right) .
$$

We begin by showing that $V^{*}$ is the unique upper semicontinuous viscosity solution of the Bellman equation for the problem associated with (1.1)(1.2). First we repeat the definition of u.s.c viscosity solution introduced in [2]. For a general Hamilton-Jacobi-Bellman equation we have the hamiltonian $H:[0, T] \times R^{n} \times R^{n} \rightarrow R^{1}$. For the optimal control problem at hand our hamiltonian is given by

$$
H(t, x, p)=\max _{y \in Y}\{p \cdot f(t, x, y)\} .
$$

Definition 1. An u.s.c real-valued function $u:[0, T] \times R^{n} \rightarrow R^{1}$ that is bounded above is an u.s.c viscosity solution of the Hamilton-Jacobi-Bellman equation

$$
u_{t}+H\left(t, x, D_{x} u\right)=0 \quad \text { on }(0, T) \times R^{n}
$$

if for any function $\phi \in C^{1}\left((0, T) \times R^{n}\right)$ for which $u-\phi$ achieves a maximum at the point $(s, y) \in(0, T) \times R^{n}$ we have that

$$
\phi_{t}(s, y)+H\left(s, y, D_{x} \phi\right)=0 \text { at }(s, y) .
$$

The sense in which upper semicontinuous terminal data is achieved for the Cauchy problem is the following, also from [2]:

Definition 2. Let $g$ be an upper semicontinuous function on $R^{n}$ and let $u$ be an upper semicontinuous function on $(0, T) \times R^{n}$. Then $u(T, x)=g(x)$ is defined as

$$
g(x)=\sup \left\{\limsup _{k \rightarrow \infty} u\left(t_{k}, x_{k}\right) \mid t_{k} \nearrow T, x_{k} \rightarrow x\right\} .
$$

For applications, approximation by continuous functions is very useful. The next theorem from [2] applies to general hamiltonians $H(t, x, p)$ that are convex in $p$ and in particular, hold for the hamiltonian of our problem in (1.3). 
Theorem 3. Under the assumption (A), there exists a unique u.s.c viscosity solution $V$ of the Hamilton-Jacobi-Bellman equation achieving the terminal data $g$. Furhtermore, if $\left\{g_{i}\right\}_{i}$ is any sequence of continuous functions on $R^{n}$ such that $g_{i} \searrow g$ as $i \rightarrow \infty$ and if $V_{i}$ is the unique continuous viscosity solution of (1.4) with $V_{i}(T, x)=g_{i}(x), i=1,2, \ldots$, then $V_{i} \searrow V$ as $i \rightarrow \infty$.

Now we prove that the relaxed value function is a u.s.c viscosity solution of (1.4) with hamiltonian (1.3).

Theorem 4. Let (A) hold. Then $V^{*}$ is the u.s.c viscosity solution of (1.3) and $V^{*}(T, x)=g(x)$.

Proof. We must first show that $V^{*}$ is u.s.c. Let $(t, x) \in(0, T) \times R^{n}$ be fixed and choose a sequence $\left\{\left(t_{i}, x_{i}\right)\right\} \subset(0, T) \times R^{n}$ such that $\left(t_{i}, x_{i}\right) \rightarrow(t, x)$ as $i \rightarrow \infty$. For each $i=1,2, \ldots$ there exists an optimal relaxed control $\mu_{i} \in Y^{*}\left[t_{i}, T\right]$ (extended as 0 to all of $[0, T]$ ) and corresponding relaxed trajectory $\xi_{i}^{*}(\cdot)$ on $\left[t_{i}, T\right]$, with $\xi_{i}^{*}\left(t_{i}\right)=x_{i}$. Then there is a subsequence with the same notation such that

$$
\mu_{i} \rightarrow \mu \text { and } \xi_{i}^{*}(T) \rightarrow \xi^{*}(T) \text { as } i \rightarrow \infty,
$$

where $\mu \in Y^{*}[t, T]$ and $\xi^{*}(\cdot)$ is the relaxed trajectory on $[t, T]$ corresponding to $\mu$ with $\xi^{*}(t)=x$ (c.f. [7, Lemma 6.2.1] for complete details).

From the upper semicontinuity of $g$, we obtain

$$
\begin{aligned}
\limsup _{i \rightarrow \infty} V^{*}\left(t_{i}, x_{i}\right) & =\limsup _{i \rightarrow \infty} g\left(\xi_{i}^{*}(T)\right) \\
& \leq g\left(\xi^{*}(T)\right) \leq V^{*}(t, x) .
\end{aligned}
$$

Next we show that $V^{*}$ is the u.s.c viscosity solution of the Bellman equation. For this purpose, let $\left\{g_{i}\right\}$ be a sequence of continuous functions such that $g_{i} \searrow g$ as $i \rightarrow \infty$. Let $V_{i}$ be the value function and $V_{i}^{*}$ the relaxed value function for the continuous terminal data $g_{i}, i=1,2, \ldots$. It is well known (see, for example $[3,7]$ ) that $V_{i}=V_{i}^{*}$ for continous data. Furthermore, $V_{i}$ is the unique continuous viscosity solution of the Bellman equation with terminal data $g_{i}$.

Since $g \leq g_{i}, \forall i$ we immediately have that $V^{*} \leq V_{i}^{*}$. By comparison theorems for continuous viscosity solutions [4] we also know that $V_{i}^{*} \geq V_{i+1}^{*}$, $i=1,2, \ldots$. Therefore

$$
V^{*} \leq \lim _{i \rightarrow \infty} V_{i}^{*}
$$

On the other hand, there exists for each $i=1,2, \ldots$ an optimal relaxed control $\mu_{i}^{*} \in Y^{*}[t, T]$ for which $V_{i}^{*}(t, x)=g_{i}\left(\xi_{i}^{*}(T)\right)$, where $\xi_{i}^{*}$ is the relaxed trajectory for $\mu_{i}^{*}$ with $\xi_{i}^{*}(t)=x$. Then, as before, there is a relaxed control $\mu^{*}$ with associated relaxed trajectory $\xi^{*}(\cdot)$ on $[t, T], \xi^{*}(t)=x$, and a subsequence, denoted again as $\left\{\mu_{i}^{*}\right\}$ such that

$$
\mu_{i}^{*} \rightarrow \mu^{*} \text { in } L^{\infty}([t, T] ; M(Y)), \quad \text { and } \xi_{i}^{*} \rightarrow \xi^{*} \text { uniformly on }[t, T]
$$


as $i \rightarrow \infty$. Then

$$
\begin{aligned}
V_{i}^{*}(t, x) & =g_{i}\left(\xi_{i}^{*}(T)\right) \\
& =g_{i}\left(\xi_{i}^{*}(T)\right)-g\left(\xi^{*}(T)\right)+g\left(\xi^{*}(T)\right) \\
& \leq g_{i}\left(\xi_{i}^{*}(T)\right)-g\left(\xi^{*}(T)\right)+V^{*}(t, x) .
\end{aligned}
$$

Given $\varepsilon>0$ fix $k>0$ such that $0 \leq g_{k}\left(\xi^{*}(T)\right)-g\left(\xi^{*}(T)\right)<\varepsilon$. Since $\left\{g_{i}\right\}$ is nonincreasing, for any fixed $k$ if $i \geq k, g_{i}\left(\xi_{i}^{*}(T)\right) \leq g_{k}\left(\xi_{i}^{*}(T)\right)$. Thus,

$$
\underset{i \rightarrow \infty}{\lim \sup _{i}} g_{i}\left(\xi_{i}^{*}(T)\right) \leq \underset{\substack{i \rightarrow \infty \\ i \geq k}}{\lim \sup _{k}} g_{k}\left(\xi_{i}^{*}(T)\right)=g_{k}\left(\xi^{*}(T)\right) \leq g\left(\xi^{*}(T)\right)+\varepsilon .
$$

Combining (1.5)-(1.8) we obtain that

$$
\lim _{i \rightarrow \infty} V_{i}^{*}(t, x)=V^{*}(t, x) .
$$

Finally, from (1.9) and Theorem 3, we conclude that $V^{*}$ is the unique u.s.c viscosity solution of the Bellman equation.

Next we want to prove that the upper semicontinuous envelope of the value function coincides with the relaxed value function.

Theorem 5. Let (A) hold and let $\widehat{V}$ denote the upper semicontinuous envelope of $V$, i.e., $\widehat{V}(t, x)=\lim \sup _{(s, y) \rightarrow(t, x)} V(s, y)$. Then $\widehat{V}=V^{*}$ on $[0, T] \times R^{n}$.

Proof. We note that since $Y[t, T] \subset Y^{*}[t, T]$ in the sense that every ordinary control $\eta \in Y[t, T]$ is a relaxed control concentrated at $\eta$, it is immediate that $V^{*} \geq V$ on $[0, T] \times R^{n}$. Since $V^{*}$ is u.s.c this implies that $V^{*} \geq \widehat{V}$.

For the other inequality, let $\left\{g_{i}\right\}_{i}$ denote a sequence of continuous functions on $R^{n}$ that converge monotonically decreasing to $g$, and let $V_{i}$ be the value function for the function $g_{i}, i=1,2, \ldots$. Let $\varepsilon>0$ and choose, for each $i=1,2, \ldots$ a control function $\eta_{i} \in Y[t, T]$ such that

$$
V_{i}(t, x) \leq g_{i}\left(\xi_{i}(T)\right)+\varepsilon,
$$

where $\xi_{i}(\cdot)$, for each $i$, is the trajectory corresponding to $\eta_{i}$ through the initial point $(t, x)$. We now make use of an argument due to Barles and Perthame [1]. We know that (at least a subsequence of) $\left\{\xi_{i}(T)\right\}_{i}$ converges to some point, say $\beta \in R^{n}$ as $i \rightarrow \infty$. Consider the solution, $\gamma_{i}(\cdot)$, of the terminal value problem

$$
\gamma_{i}(\tau)=\beta+\int_{\tau}^{T} f\left(s, \gamma_{i}(s), \eta_{i}(s)\right) d s, \quad t \leq \tau \leq T .
$$

For each $i, \gamma_{i}(T)=\beta$, i.e., the trajectories $\gamma_{i}$ all end at the fixed point $\beta$. Now we trace them back to see where they start at time $t$. Set $\gamma_{i}(t)=\kappa_{i}$, $i=1,2, \ldots$ and consider the equivalent trajectories which we label $\delta_{i}(\cdot)$ given by

$$
\delta_{i}(\tau)=\kappa_{i}+\int_{t}^{\tau} f\left(s, \delta_{i}(s), \eta_{i}(s)\right) d s, \quad t \leq \tau \leq T
$$


That is, $\gamma_{i}(\cdot) \equiv \delta_{i}(\cdot)$. From condition (A) and standard results in o.d.e.s we have that

$$
\sup _{t \leq \tau \leq T}\left|\xi_{i}(\tau)-\delta_{i}(\tau)\right| \leq K\left|\xi_{i}(T)-\beta\right|
$$

Consequently, at $\tau=t$,

$$
\left|x-\kappa_{i}\right| \leq K\left|\xi_{i}(T)-\beta\right| \rightarrow 0 \text { as } i \rightarrow \infty .
$$

Then we have that

$$
\begin{aligned}
V_{i} \leq g_{i}\left(\xi_{i}(T)\right)+\varepsilon & =g_{i}\left(\xi_{i}(T)\right)-g\left(\delta_{i}(T)\right)+g\left(\delta_{i}(T)\right)+\varepsilon \\
& \leq g_{i}\left(\xi_{i}(T)\right)-g\left(\delta_{i}(T)\right)+V\left(t, \kappa_{i}\right)+\varepsilon \\
& =g_{i}\left(\xi_{i}(T)\right)-g(\beta)+V\left(t, \kappa_{i}\right)+\varepsilon .
\end{aligned}
$$

From the facts $\xi_{i}(T) \rightarrow \beta, g_{i} \searrow g$, as $i \rightarrow \infty$ we have, using the argument leading to (1.8), that $\lim \sup _{i \rightarrow \infty} g_{i}\left(\xi_{i}(T)\right) \leq g(\beta)$. Further, $V\left(t, \kappa_{i}\right) \leq$ $\widehat{V}\left(t, \kappa_{i}\right)$. Combining these facts, we now use the upper semicontinuity of $\widehat{V}$, (1.10), and (1.11) to see that

$$
V^{*}(t, x)=\limsup _{i \rightarrow \infty} V_{i} \leq \widehat{V}(t, x)+\varepsilon,
$$

so that $V^{*} \leq \widehat{V}$ and we are done.

\section{REFERENCES}

1. G. Barles and B. Perthame, Discontinuous solutions of deterministic optimal stopping time problems, Math. Modelling Numer. Anal. 21 (1987), 557-579.

2. E. N. Barron and R. Jensen, Semicontinuous viscosity solutions for Hamilton-Jacobi equations with convex Hamiltonian, Comm. Partial Differential Equations 15 (1990), 17131742.

3. L. Cesari, Optimization-theory and applications, Springer-Verlag, New York, 1983.

4. M. G. Crandall and P.-L. Lions, Viscosity solutions of Hamilton-Jacobi equations, Trans. Amer. Math. Soc. 282 (1984), 1-42.

5. H. Ishii, Perron's method for Hamilton-Jacobi equations, Duke Math. J. 55 (1987), 369384.

6. N. N. Krasovskii and A. I. Subbottin, Game theoretical control problems, Springer-Verlag, New York, 1988.

7. J. Warga, Optimal control on differential and functions equations, Academic Press, New York, 1972.

Department of Mathematical Sciences, Loyola University of Chicago, Chicago, ILLINOIS 60626 